\title{
DETERMINAÇÃO ELETROQUÍMICA DA CAPACIDADE ANTIOXIDANTE PARA AVALIAÇÃO DO EXERCÍCIO FÍSICO
}

\author{
Paulo Guimarães Gandra, Armindo Antônio Alves e Denise Vaz de Macedo* \\ Departamento de Bioquímica, Instituto de Biologia, Universidade Estadual de Campinas, CP 6109, 13083-970 Campinas - SP \\ Lauro Tatsuo Kubota \\ Departamento de Química Analítica, Instituto de Química, Universidade Estadual de Campinas, CP 6154, 13084-971 Campinas - SP
}

Recebido em 26/9/03; aceito em 19/5/04; publicado na web em 9/9/04

\begin{abstract}
ELECTROCHEMICAL DETERMINATION OF ANTIOXIDANT CAPACITY FOR PHYSICAL EXERCISE EVALUATION. Physical training can adapt or cause injury to skeletal muscles implicating metabolic alterations, which can be detected by biochemical analysis. Apparently the increase in the production of reactive oxygen species (ROS) is involved in both processes. Enzymatic and low molecular weight antioxidants (LMWA) minimize ROS's deleterious action through redox reactions. Cyclic voltammetry $(\mathrm{CV})$ has been suggested as a tool to quantify the antioxidant capacity conferred by LMWA. The use of CV to evaluate the modulation of the antioxidant capacity conferred by LMWA in response to physical exercise is discussed here.
\end{abstract}

Keywords: cyclic voltammetry; antioxidant capacity; physical exercise.

\section{INTRODUÇÃO}

Os músculos esqueléticos geram continuamente uma cascata de intermediários metabólicos de baixo peso molecular, difusíveis, derivados da redução monoeletrônica do oxigênio. Esses intermediários são conhecidos como espécies reativas de oxigênio (EROs) e incluem um amplo espectro de espécies radicalares, como o radical ânion superóxido $\left(\mathrm{O}_{2}^{-*}\right)$ e o radical hidroxila $\left(\mathrm{OH}^{-}\right)$e não radicalares como o peróxido de hidrogênio $\left(\mathrm{H}_{2} \mathrm{O}_{2}\right)$. $\mathrm{O}$ músculo esquelético também produz radical óxido nítrico ( $\mathrm{NO}^{*}$ ), pela reação catalisada pela enzima óxido nítrico sintase, a partir do aminoácido arginina ${ }^{1,2}$. O NO*, por sua vez, pode reagir com $\mathrm{O}_{2}^{-\bullet}$ formando peroxinitrito $\left(\mathrm{ONOO}^{-}\right)$, um intermediário instável, de alta reatividade ${ }^{2}$.

Todas essas espécies exibem, em menor ou maior grau, atividade redox, podendo influenciar processos intracelulares importantes, sensíveis a esse estado. Uma vez formados, não escolhem seus alvos, oxidando lipídios, proteínas, ácidos nucléicos ou quaisquer outras biomoléculas que estiverem próximas a eles. A atividade oxidante das EROs, no entanto, é limitada por antioxidantes enzimáticos e não enzimáticos, além de outras proteínas e enzimas ${ }^{3-5}$. A Figura 1 ilustra os mecanismos de produção de EROs e dos sistemas de defesa antioxidante.

A sobrevivência celular frente ao ataque dos radicais livres depende, portanto, do equilíbrio entre os processos de produção e de eliminação das EROs. Qualquer circunstância que desequilibre estes dois processos, tal como exercício físico, envelhecimento ou patologias (cânceres, diabete, arteriosclerose, entre outras), pode induzir a instalação de uma condição chamada estresse oxidativo, em que prevalece a formação de oxidantes aos antioxidantes ${ }^{6}$. Nesse caso, o acúmulo de reações de oxidação pode induzir alterações severas em várias estruturas celulares ${ }^{7}$. Uma das hipóteses para explicar o aumento nos níveis de lesão muscular induzida pelo exercício físico propõe que a perda de integridade da membrana celular ocorra principalmente como consequiência de um ataque das EROs ${ }^{8}$.

*e-mail: labex@unicamp.br

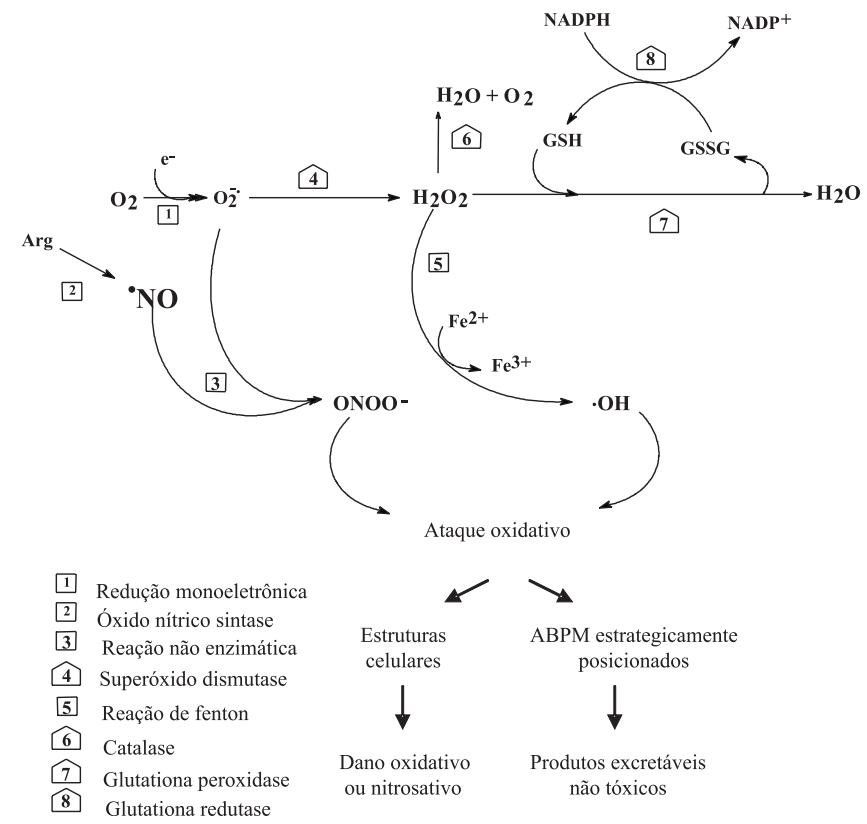

Figura 1. Mecanismos de ataque de EROs, partindo da redução monoeletrônica do $\mathrm{O}_{2}$ e os sistemas de defesa antioxidante. O símbolo $\square$ refere-se a mecanismos de produção de EROs e o símbolo $\square$, às principais enzimas da defesa antioxidante

\section{DEFESA ANTIOXIDANTE}

O sistema antioxidante não enzimático é constituído principalmente pelos antioxidantes de baixo peso molecular (ABPM), que desativam diretamente as EROs. Os ABPM podem ser sintetizados endogenamente, ser restos metabólicos, ou advir da alimentação. Estão presentes nos organismos em número e concentração muito maior que os antioxidantes enzimáticos. Como os ABPM podem ser hidrofílicos ou lipofílicos, estão presentes nos locais específicos em que ocorrem os danos causados por estresse oxidativo, exercendo, dessa 
forma um papel fundamental para a capacidade antioxidante total de sistemas biológicos ${ }^{5}$. A glutationa reduzida (GSH), o ácido úrico e a vitamina $C$ são exemplos de ABPM hidrofílicos. A bilirrubina, o $\beta$ caroteno e a vitamina E são exemplos de ABPM lipofílicos.

Variações das concentrações de ABPM no plasma e tecido podem ocorrer em decorrência do treinamento físico ou da realização de sessões extenuantes de exercício físico. Alguns estudos analisam a variação de ABPM isoladamente ${ }^{9}$, outros analisam a variação da capacidade antioxidante total do plasma e tecidos. Por exemplo, Child e colaboradore ${ }^{10}$ observaram aumento na capacidade antioxidante total do plasma após a realização de corrida de meia maratona em indivíduos treinados, com concomitante aumento em indicadores de peroxidação lipídica e de lesão muscular. Em um outro estudo de Child e colaboradores $^{11}$, envolvendo a realização de exercício excêntrico em humanos, a capacidade antioxidante total do plasma não se mostrou alterada, enquanto a do músculo mostrou aumentos significativos. Liu e colaboradores ${ }^{12}$ mostraram aumentos na capacidade antioxidante total e concentração de ácido úrico e manutenção das concentrações de vitamina $\mathrm{C}, \alpha$-tocoferol, $\beta$-caroteno e retinol plasmático em corredores treinados, após participação em maratona. Brites e colaboradores ${ }^{13}$ relataram que jogadores de futebol apresentavam uma capacidade antioxidante total do plasma $25 \%$ maior que aquela de indivíduos sedentários, além de concentrações plasmáticas maiores de ácido ascórbico, ácido úrico e $\alpha$-tocoferol, indicando uma adaptação da defesa antioxidante nos indivíduos treinados.

Venditti e Di $\mathrm{Meo}^{14}$ observaram que ratos treinados apresentavam maior capacidade antioxidante total do fígado, coração e músculos que os ratos controle, sendo que os grupos controle e treinado mostraram aumentos nos níveis de lesão de tecidos após a realização de uma sessão de exercício até a exaustão. Os autores justificaram a ocorrência de lesão no grupo treinado, apesar da adaptação do sistema de defesa antioxidante, devido ao tempo para exaustão para este grupo ser muito maior que para o grupo controle, sugerindo que os antioxidantes contribuiriam para a melhora no desempenho.

Além do sistema não-enzimático, pelo menos três enzimas específicas para EROs são expressas constitutivamente pelos músculos e degradam seletivamente espécies moleculares individuais. A superóxido dismutase (SOD) dismuta o $\mathrm{O}_{2}^{-\cdot}$, enquanto a catalase (CAT) e a glutationa peroxidase (GPX) consomem o $\mathrm{H}_{2} \mathrm{O}_{2}{ }^{15,16}$. A enzima glutationa redutase (GR) trabalha em sintonia com a GPX, pois é a enzima responsável pela redução do GSSG formado na reação da GPX a GSH, às custas de NADPH oriundo, principalmente, da via das pentoses fosfato, imprescindível para a ação da GPX ${ }^{15}$.

A literatura aponta tanto aumento quanto diminuição na atividade das enzimas da defesa antioxidante, dependendo do protocolo de exercício utilizado ${ }^{3,15,17,18}$. Esses dados reforçam que a atividade e a expressão dessas enzimas parecem ser moduladas pela concentração de EROs produzida durante os exercícios ${ }^{17,18}$. Resultados recentes em nosso laboratório mostraram que as enzimas CAT E GR eritrocitárias têm suas atividades significativamente diminuídas, em paralelo com um aumento na concentração da proteína de estresse HSP72 em leucócitos, quando ratos treinados são submetidos a um protocolo de corrida em esteira com aumento no número de sessões diárias de exercícios ${ }^{19}$. Ou seja, aparentemente há um grande aumento nos níveis de estresse oxidativo com a conseqüente diminuição no tempo de recuperação dos animais, que se relaciona com a perda das adaptações positivas alcançadas com o treinamento.

\section{ESTADO REDOX CELULAR E ADAPTAÇÃO AO EXERCÍCIO}

Como a geração de EROs no exercício físico parece estar associada tanto ao sucesso quanto ao fracasso adaptativo ao treinamento, na metade da década de 90, começou a ser proposto com maior ênfase na literatura que as EROs seriam capazes de produzir um efeito bifásico sobre as funções contráteis de músculos esqueléticos não fadigados ${ }^{16,20}$. Mostrou-se que baixos níveis de EROs, presentes em condições basais, eram essenciais para a produção normal de força. Um aumento modesto na produção de EROs aumentava a força produzida, sendo que este efeito positivo era revertido em altas concentrações de EROs de maneira tempo e dose-dependente. Da mesma forma, os efeitos negativos eram inibidos pelo pré-tratamento do músculo com antioxidantes, tais como as enzimas CAT e SOD, e revertidos pela administração de agentes redutores, tal como o ditiotreitol $^{21}$.

Todas essas observações levaram à proposição do modelo homeostático do estado redox celular. Este modelo assume que o estado redox citosólico é uma variável regulada fisiologicamente, sendo balanceado pela produção de oxidantes e capacidade de tamponamento antioxidante, predizendo que existe um estado redox intracelular que é ótimo para a geração de força.

Pelo exposto, fica claro que existe uma separação tênue entre um estado de adaptação positiva e um estado de ruptura do processo adaptativo, que necessariamente induz queda no rendimento físico. Dessa forma, um dos principais desafios atualmente no desenvolvimento de metodologias de treinamento específicas para as diferentes modalidades esportivas está em propiciar ao organismo um nível ótimo de condicionamento, com a realização de esforços mais intensos, com um custo energético menor e sem a prevalência de lesões. A adequação de intensidade $x$ quantidade de exercício $x$ tempo de recuperação desse esforço parece ser a grande responsável por ambos os fenômenos.

Nosso laboratório trabalha com a hipótese de que o monitoramento conjunto da atividade da defesa antioxidante com os níveis de lesão muscular e ataque oxidativo de tecidos ou fluidos corpóreos poderia permitir interferir nas cargas de treino aplicadas, quando necessário ${ }^{22}$. Para esta finalidade, além da quantificação das atividades das enzimas antioxidantes nas células sanguíneas é imprescindível a existência de bons indicadores da capacidade antioxidante total de fluidos ou tecidos biológicos, uma vez que a determinação individual das concentrações de cada ABPM é praticamente inviável.

\section{DETERMINAÇÃO DA CAPACIDADE ANTIOXIDANTE CONFERIDA POR ABPM}

A literatura apresenta várias metodologias para a dosagem da atividade antioxidante total do plasma ${ }^{23,24}$. Alguns autores afirmam que a vantagem sobre a dosagem de cada antioxidante separadamente é que a determinação da capacidade antioxidante total leva em conta o sinergismo entre todos os componentes do sistema, já que ele é bastante complexo ${ }^{23,25}$. Alguns métodos, como o de Wayner e colaboradores $^{26}$, determinam o tempo de consumo de todos os antioxidantes da amostra quando ela é exposta a radicais produzidos a uma velocidade conhecida. Neste caso, a capacidade antioxidante representa a concentração de radicais que são degradados, refletindo, indiretamente, a quantidade de antioxidantes da amostra. Normalmente utiliza-se o TROLOX (2-carboxi-2,5,7,8-tetrametil-6cromanol), um análogo hidrossolúvel da vitamina $\mathrm{E}$ como referência, sendo o resultado dado em capacidade antioxidante equivalente ao TROLOX. Esta metodologia foi modificada por alguns autores, que utilizaram diferentes técnicas e fontes de radicais para o monitoramento do consumo dos antioxidantes ${ }^{27}$. Outras metodologias registram a capacidade da amostra tamponar radicais, prevenindo danos a determinadas macromoléculas ${ }^{25,28}$.

A Tabela 1 resume as principais características de alguns métodos descritos para a dosagem da capacidade antioxidante total. 
Tabela 1. Principais características de alguns métodos para a dosagem de capacidade antioxidante total

\begin{tabular}{|c|c|c|c|c|}
\hline Ref. & Princípio & $\begin{array}{l}\text { Fonte Geradora } \\
\text { de Radicais }\end{array}$ & Métodos de Análise & Comentários \\
\hline 26 & $\begin{array}{l}\text { Determina o tempo de consumo } \\
\text { dos antioxidantes expostos a radicais } \\
\text { produzidos a uma taxa conhecida, } \\
\text { através da determinação da peroxidação } \\
\text { lipídica }\end{array}$ & ABAP & Eletrodo de Oxigênio & $\begin{array}{l}\text { Demorado, o eletrodo de oxigênio não } \\
\text { se mantém estável pelo tempo } \\
\text { necessário }^{23}\end{array}$ \\
\hline 27 & $\begin{array}{l}\text { Determina o tempo de consumo dos } \\
\text { antioxidantes pela oxidação de luminol }\end{array}$ & ABAP & Quimioluminescência & $\begin{array}{l}\text { Exige menor tempo do que o método } \\
\text { proposto por Wayner et al. } .^{26}\end{array}$ \\
\hline 29 & $\begin{array}{l}\text { Determina a capacidade da amostra } \\
\text { tamponar radicais peroxil, prevenindo } \\
\text { danos a uma proteína hidrossolúvel } \\
\text { (R ou B-Ficoeritrina) de propriedades } \\
\text { fluorescentes }\end{array}$ & AAPH & Fluorimetria & $\begin{array}{l}\text { O alto custo da proteína encarece o } \\
\text { método }\end{array}$ \\
\hline 30 & $\begin{array}{l}\text { Utiliza as propriedades fluorescentes } \\
\text { das ficoeritrinas }\end{array}$ & ABAP & Fluorimetria & $\begin{array}{l}\text { O tempo para uma única análise pode } \\
\text { ser de até } 70 \mathrm{~min}\end{array}$ \\
\hline 31 & $\begin{array}{l}\text { Quantifica a descoloração de cátions } \\
\text { radicais ABTS }{ }^{*+} \text { pré-formados, que irão } \\
\text { se reduzir após adição de antioxidantes }\end{array}$ & $\mathrm{ABTS}^{*+}$ & Espectrofotometria & $\begin{array}{l}\text { O resultado obtido é fortemente } \\
\text { influenciado pelo tempo de medida }\end{array}$ \\
\hline 32 & $\begin{array}{l}\text { Mede a redução do } \mathrm{Fe}^{3+} \text { (na forma do } \\
\text { complexo ferro-tripiridiltriazina) para a } \\
\text { forma ferrosa, que desenvolve uma cor } \\
\text { azul intensa }\end{array}$ & Não utiliza radicais & Espectrofotometria & Não é sensível a proteínas \\
\hline
\end{tabular}

Na metodologia descrita por Glazer ${ }^{29}$ utiliza-se a propriedade fluorescente de uma proteína, determinando a perda de sua fluorescência pelo ataque de radicais. Partindo da metodologia de Glazer $^{29}$, outros autores elaboraram métodos que se baseiam nos mesmos princípios do método original, como Ghiselli e colaboradores $^{25}$ e Cao e colaboradores ${ }^{30}$.

Em relação às diferentes metodologias existentes para determinação da capacidade antioxidante total, podemos ainda diferenciálas pela informação que podem fornecer. Ao se medir o tempo necessário para se depletar a atividade antioxidante de uma amostra quando radicais são produzidos a uma taxa conhecida, apenas se quantificam os antioxidantes e não a sua reatividade ${ }^{26,27}$; já quando se determina a porcentagem de inibição de radicais por um certo período de tempo, além da inibição total causada pela amostra, podese inferir quanto à reatividade e quantidade de antioxidantes ${ }^{30}$.

\section{VOLTAMETRIA CÍCLICA}

A voltametria cíclica (VC) é utilizada normalmente para estudar a transferência de elétrons entre moléculas e eletrodos. Uma vez que os ABPM podem agir doando elétrons diretamente às EROs, funcionando como agentes redutores, a avaliação do potencial de oxidação de uma amostra biológica através da VC pode refletir sua capacidade antioxidante.

Para a realização da VC, utiliza-se uma cela com três eletrodos. Os eletrodos são conectados a um potenciostato, controlado por um microcomputador, que irá aplicar uma diferença de potencial entre o eletrodo de trabalho e o eletrodo de referência, a uma taxa constan$\mathrm{te}^{33}$. O eletrodo auxiliar irá prover a corrente para o eletrodo de trabalho, de forma que praticamente não passe nenhuma corrente pelo eletrodo de referência, possibilitando que o seu potencial se mantenha constante ${ }^{33}$. Na VC é aplicado um potencial inicial que varia linearmente até um potencial limite, no qual inverte-se o sentido da varredura para a direção do potencial inicial ${ }^{33,34}$. Para o estudo de agentes redutores, o potencial deve ser aplicado no sentido do negativo para o positivo, apenas sendo analisada a corrente anódica. A curva obti- da pela variação da corrente (eixo y) versus a variação de potencial (eixo x) compõe o voltamograma. Dessa forma, os antioxidantes transferem seus elétrons a um potencial específico para o eletrodo de trabalho, que se apresenta com carga positiva, registrando picos de corrente anódica no voltamograma. Quando a região próxima da superfície do eletrodo de trabalho está escassa de compostos passíveis de se oxidarem, observa-se a diminuição da corrente ${ }^{33,34}$.

A escolha do tipo de eletrodo de trabalho depende da faixa de potencial que se pretende estudar. Eletrodos de carbono vítreo, que atuam no intervalo de $-0,5 \mathrm{~V}$ a $+1,1 \mathrm{~V}$ contra o eletrodo de $\mathrm{Ag} / \mathrm{AgCl}$ são adequados para determinar a capacidade antioxidante de amostras biológicas.

O poder redutor de uma amostra é dado em função de dois parâmetros. O potencial de pico (Ep) anódico, determinado pelo potencial redox do composto analisado, e o valor de corrente de pico. Quanto menor o valor de Ep de uma amostra, maior é sua capacidade de doar elétrons. No caso de amostras biológicas, diferentes compostos com Ep próximos podem causar a sobreposição de picos de corrente anódica, implicando na formação de um pico composto por diferentes antioxidantes, que apresentará um valor de Ep característico, dependente da concentração de cada antioxidante que compõe o pico. $\mathrm{O}$ potencial de pico também pode ser representado por $\mathrm{E}_{1 / 2}$, que representa o potencial no ponto em que a corrente apresenta metade do seu valor de pico o que, segundo alguns autores, pode ser mais preciso que a determinação de $\mathrm{Ep}^{33}$.

O valor da corrente de pico (Ip) de corrente anódica é dependente da concentração de antioxidantes que compõem cada pico de corrente anódica. A aplicação da primeira lei de difusão de Fick (Equação 1) demonstra que as variações dos valores da corrente de pico são diretamente proporcionais às variações das concentrações dos antioxidantes.

$\mathrm{i}_{\mathrm{t}}=\mathrm{nFAD}_{0}\left(\partial \mathrm{C}_{0} / \partial_{\mathrm{x}}\right), \quad \mathrm{x}=0, \mathrm{t}$

onde $\mathrm{i}_{\mathrm{t}}$ é o valor de corrente por tempo; $\mathrm{n}$, o número de elétrons; $\mathrm{F}$, a constante de Faraday; A, a área do eletrodo; $\mathrm{C}_{0}$, a concentração de 
compostos que podem ser oxidados; $\mathrm{D}_{0}$, o coeficiente de difusão das espécies reduzidas; $\mathrm{t}$, tempo e $\mathrm{x}$, a distância da superfície do eletrodo.

Para a determinação do valor de Ip pode ser usado como linha de base o voltamograma do tampão utilizado na amostra ${ }^{33}$, ou uma reta unindo os valores de mínima do pico ${ }^{35}$ como demonstrado na Figura 2.

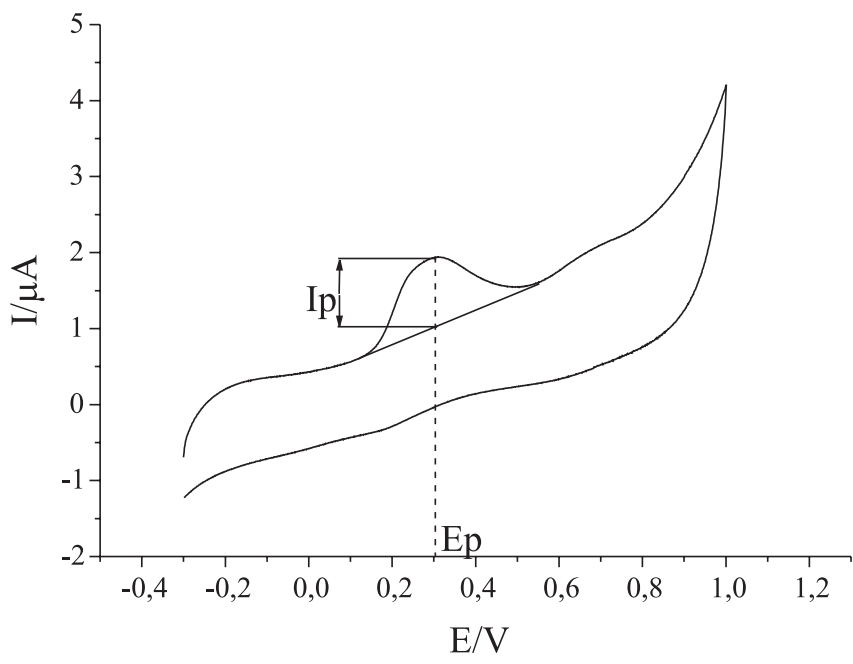

Figura 2. Voltamograma típico de plasma de indivíduo jovem saudável, realizado em uma faixa de potencial de $-0,3$ a 1,0 $\mathrm{V}$

Dessa forma, Galato e colaboradores ${ }^{36}$ determinaram a capacidade antioxidante de compostos fenólicos e outros compostos relacionados, em comparação com a espectroscopia e potenciometria. Os autores observaram que quanto maior o número de hidroxilas ligadas ao anel aromático dos compostos analisados, maior era sua atividade antioxidante. Tan e colaboradore ${ }^{37}$ estudaram a capacidade antioxidante de uma amina, a $\mathrm{N}^{1}$-acetil- $\mathrm{N}^{2}$-formil-5-metoxicinuramina, metabólito da via de degradação da melatonina e mostraram, com o uso da $\mathrm{VC}$, que esta amina é oxidada via dois elétrons em duas etapas monoeletrônicas $(\mathrm{Ep}(\mathrm{s})=456$ e $668 \mathrm{mV})$, diferente de outros antioxidantes, que doam somente um elétron. Esses dados, em conjunto com a proteção conferida por esta amina a danos in vitro ao DNA e peroxidação lipídica de homogenatos de fígado, demonstraram que esta molécula apresenta uma grande capacidade antioxidante, conferindo proteção a neurônios hipocampais. A Tabela 2 apresenta valores de potenciais de pico de diferentes antioxidantes, determinados através da VC.
A VC permite a quantificação dos ABPM hidrofílicos e lipofílicos dentro de uma faixa de concentração de 1 a $10 \mu \mathrm{M}$, adequada às concentrações de compostos antioxidantes encontradas em amostras biológicas $^{39,40}$. Os volumes mínimos de amostras utilizadas normalmente variam de 200 a $300 \mu \mathrm{L}$, utilizando celas de 200 a $3000 \mu \mathrm{L}$. Para os ABPM hidrofílicos, dilui-se a amostra de fluido biológico na razão de 1:1 em tampão apropriado, que não apresente potencial de oxidação, como em tampão fosfato em solução salina (PBS), realizando a leitura desta solução ${ }^{39,40}$. A preparação de homogenatos de tecidos deve ser feita em PBS, e não requer posterior diluição para realização da análise voltamétrica ${ }^{39,41}$. Para determinação dos ABPM lipofílicos utilizam-se aproximadamente $4 \mathrm{~mL}$ de fluido ou homogenato de tecido diluído em PBS, e os ABPM são extraídos com uma mistura de etanol/hexano, utilizando o sobrenadante diluído em uma solução de acetonitrila com perclorato de tetrabutilamônio, que funciona como eletrólito ${ }^{39,41}$. Os componentes principais de cada pico de corrente anódica podem ser determinados por diferentes procedimentos, como o uso de HPLC com detectores eletroquímicos ${ }^{38,42}$, pela adição de enzimas, como a ascorbato oxidase e uricase $\mathrm{e}^{41}$, de maneira a relacionar a diminuição da concentração de ABPM à diminuição dos valores de Ip, assim como adição de vários antioxidantes nas amostras estudadas, com a subseqüente determinação de seus potenciais de oxidação ${ }^{38}$.

Chevion e colaboradores ${ }^{38}$ desenvolveram um procedimento utilizando a VC para determinar a capacidade antioxidante do plasma. Especificamente para essa determinação utiliza-se, normalmente, uma faixa de potencial de $-0,3 \mathrm{a}+1,3 \mathrm{~V}$, a uma velocidade de $100 \mathrm{mV} / \mathrm{s}$, um eletrodo de trabalho de carbono vítreo de aproximadamente $3 \mathrm{~mm}$ de diâmetro, um eletrodo de platina como auxiliar e outro de $\mathrm{Ag} / \mathrm{AgCl}$ como controle ${ }^{38-40}$. O plasma, normalmente, apresenta um pico de corrente anódica principal em potencial de $400 \pm 50 \mathrm{mV}$, principalmente relativo à oxidação de ácido ascórbico e ácido úrico ${ }^{38,40,41}$, como ilustrado na Figura 2. O segundo pico é encontrado a um potencial de $920 \pm 25 \mathrm{mV}$, sendo provavelmente decorrente de hemólise durante a separação do plasma das células do sangue, sendo composto principalmente por NADPH das hemácias, podendo ser composto também por ácido lipóico ${ }^{39-41}$. Para extratos lipofílicos do plasma observam-se 3 picos anódicos a 490, 690 e $880 \mathrm{mV}$, relacionados, respectivamente, à oxidação da coenzima $\mathrm{Q}_{10}$ e caroteno, de tocoferóis e o terceiro pico provavelmente a uma forma oxidada de acido lipóico (possivelmente ligado a um grupo acetila) ${ }^{39}$. As características do voltamograma mostram-se iguais para o plasma desproteinizado e plasma com proteínas, indicando que a capacidade antioxidante determinada por VC reflete a capacidade de ABPM e não de outras macromoléculas como proteínas ${ }^{38}$. Além disso, a preservação do plasma a $-70{ }^{\circ} \mathrm{C}$ por 6 meses, assim como pequenas

Tabela 2. Valores de potenciais de oxidação de alguns compostos antioxidantes, determinados por VC, expressos em Ep ou Ep 1/2 $_{2}$

\begin{tabular}{|c|c|c|c|c|c|}
\hline Ref. & Composto & Tampão & $\mathrm{pH}$ & $\mathrm{Ep}(\mathrm{mV})$ & $\mathrm{Ep}_{1 / 2}(\mathrm{mV})$ \\
\hline $37^{*}$ & $\mathrm{~N}^{1}$-acetil- $\mathrm{N}^{2}$-formil-5-metoxicinuramina & PBS $(0,05 \mathrm{M})$ & 7,4 & 156 e 668 & \\
\hline $38^{*}$ & Ácido ascórbico & Fosfato $(0,2 \mathrm{M})$ & 7,4 & & 380 \\
\hline $38^{*}$ & Ácido úrico & Fosfato $(0,2 \mathrm{M})$ & 7,4 & & 420 \\
\hline $38^{*}$ & TROLOX & Fosfato $(0,2 \mathrm{M})$ & 7,4 & & 145 \\
\hline $36^{\#}$ & Ácido cafeico & Fosfato $(0,1 \mathrm{M})$ & 7,8 & 142 & \\
\hline $36^{\#}$ & Ácido ferúlico & Fosfato $(0,1 \mathrm{M})$ & 7,8 & 410 & \\
\hline $36^{\#}$ & Ácido para-coumarico & Fosfato $(0,1 \mathrm{M})$ & 7,8 & 736 & \\
\hline $36^{\#}$ & Ácido gálico & Fosfato $(0,1 \mathrm{M})$ & 7,8 & 113 & \\
\hline $36^{\#}$ & Galato de metila & Fosfato $(0,1 \mathrm{M})$ & 7,8 & 62 & \\
\hline $36^{\#}$ & Ácido lipóico & Fosfato $(0,2 \mathrm{M})$ & 7,4 & & 900 \\
\hline
\end{tabular}

$*$ eletrodo de carbono vítreo, referência $\mathrm{Ag} / \mathrm{AgCl}$ e velocidade $=100 \mathrm{mV} / \mathrm{s} ;{ }^{*}$ eletrodo de carbono vítreo, referência ECS e velocidade $=25 \mathrm{mV} / \mathrm{s}$. 
alterações no $\mathrm{pH}(7,0-7,6)$ parecem não alterar os resultados obtidos através da $\mathrm{VC}^{38,39}$.

Existem trabalhos que utilizam a VC comparando a variação da capacidade antioxidante de ABPM do plasma em função da existência de patologias e diferentes tratamentos. Chevion e colaboradores $^{43}$ pesquisaram a capacidade antioxidante do ácido lipóico, uma molécula comumente encontrada no plasma humano, encontrando uma correlação linear entre a corrente anódica obtida por VC em extrato hidrofílico a $900 \mathrm{mV}$ e a concentração desse ácido. Esses autores sugeriram que o segundo pico anódico, observado no voltamograma do plasma humano, relaciona-se à oxidação da forma mais reduzida do ácido lipóico, que possui dois grupamentos tiólicos ($\mathrm{SH}$ ). O plasma de pacientes diabéticos tratados com ácido lipóico mostrou valores maiores de Ip que de pacientes que não recebiam esse suplemento; ainda, quanto mais severo fosse o quadro de neuropatia destes pacientes sob tratamento com o ácido lipóico, menor era o valor de Ip de seu plasma.

Beit-Yannai e colaboradores ${ }^{44}$ determinaram a capacidade antioxidante dos ABPM de ratos submetidos à lesão cerebral, observando 2 picos de corrente anódica para o tecido cerebral. Após 5 min da lesão observou-se uma diminuição de $40 \%$ na capacidade antioxidante dos ABPM hidrofílicos do cérebro. Após 24 h ocorreu uma redução de $60 \%$, sendo que em 48 h, assim como após 7 dias, a capacidade antioxidante dos ABPM já estava normal.

Uma outra característica interessante da VC é permitir a determinação da capacidade antioxidante total de outros fluidos que não o plasma, como por exemplo, na saliva e em extratos de pele ${ }^{39,45}$. Ambos os métodos representam meios menos invasivos e mais práticos para se determinar o estado das defesas antioxidantes de um indivíduo. Observou-se um aumento do potencial de oxidação da saliva relacionado à idade dos doadores, da mesma forma que amostras de saliva obtidas de indivíduos portadores de diferentes patologias também mostraram alterações nos potenciais de oxidação, em relação aos indivíduos controle ${ }^{45}$. A capacidade antioxidante em extratos de pele foi determinada após incubação de PBS na superfície da pele por $30 \mathrm{~min}^{39}$. No entanto, os dois picos observados em humanos estão acima de um potencial de $500 \mathrm{mV}$, não sendo compostos nem por ácido úrico nem por ácido ascórbico, dois importantes ABPM de potenciais de oxidação menores que $500 \mathrm{mV}^{39}$.

A aplicação da VC para análises clínicas, assim como em laboratórios de pesquisa deve ser feita com certa cautela, já que os resultados podem sofrer interferências. No procedimento da VC com fluidos biológicos e homogenatos de tecidos, deve ser dada atenção especial no polimento do eletrodo de trabalho. As grandes quantidades de proteínas presentes nas amostras aderem-se na superfície do eletrodo, interferindo diretamente no resultado. $\mathrm{O}$ polimento do eletrodo deve ser feito, portanto, antes de cada análise, mantendo assim a sensibilidade do eletrodo ${ }^{38-41}$. Variáveis como a temperatura, a concentração dos tampões utilizados, os reagentes utilizados na extração da amostra biológica, o tamanho do eletrodo de trabalho e o tipo do eletrodo de referência também podem influenciar nos resultados obtidos.

\section{PERSPECTIVAS}

Tendo em vista a versatilidade da técnica, a utilização da VC é uma boa alternativa metodológica para acompanhar as modulações agudas e crônicas da capacidade antioxidante total do plasma, saliva e tecidos, conferidas pelos ABPM em resposta ao exercício físico e deverá ser motivo de intensas investigações. A voltametria cíclica mostra-se um método muito vantajoso e promissor para essas determinações. Possui baixo custo, uma vez que para cada análise todos os eletrodos e a cela são reutilizados. Sua execução é fácil e rápida, pois não requer o desenvolvimento de eletrodos especiais nem de reagentes, exigindo pouco tempo para a realização de uma leitura. Além disto, a utilização de técnicas de pulso diferencial e onda quadrada poderão aumentar ainda mais o uso da voltametria para esta finalidade.

\section{LISTA DE ABREVIAÇÕES}

ABAP- 2,2'-azobis (2-amidopropano)

AAPH- 2, 2'-azobis (2-amidinopropano) dihidrocloreto

$\mathrm{ABTS}^{+}$- radical cátion do (ácido 2,2'-azinobis-(3-etil-benzotiazolino6-sulfônico)

ABPM - antioxidantes de baixo peso molecular

AMFK- $\mathrm{N}^{1}$-acetil- $\mathrm{N}^{2}$-formil-5- metoxicinuramina

EROs - espécies reativas de oxigênio

CAT - catalase

Ep - potencial de pico

$\mathrm{Ep}_{1 / 2}-$ potencial em que a corrente representa metade do valor de pico

GSH - glutationa reduzida

GSSG - glutationa oxidada

GR - glutationa redutase

GPX - glutationa peroxidase

HPLC - cromatografia líquida de alta eficiência

Ip - corrente de pico

NADPH- nicotonamida adenina dinucleotídeo fosfato

PBS- tampão fosfato em solução salina

SOD - superóxido dismutase

TROLOX - 2-carboxi-2,5,7,8-tetrametil-6-cromanol

VC - voltametria cíclica

\section{REFERÊNCIAS}

1. Tews, D. S.; Goebel, H. H.; Clin. Immunol. Immunopathol. 1998, 87, 240; Frandsen, U.; Lopez-Figueroa, M.; Hellsten, Y.; Biochem. Biophys. Res. Commun. 1996, 227, 88.

2. Reid, M. B.; Acta Physiol. Scand. 1998, 162, 401.

3. Smolka, M. B.; Zoppi, C. C.; Alves, A. A.; Silveira L. R.; Marangoni, S.; Pereira-da-Silva, L.; Novello, J. C.; Macedo, D.V.; Am. J. Physiol. Regul. Integr. Comp. Physiol. 2000, 279, R1539.

4. Reid, M. B.; Durham, W. J.; Ann. N. Y. Acad. Sci. 2002, 959, 108.

5. Halliwell, B.; Gutteridge, J. M. C. Em Free Radicals in Biology and Medicine, Halliwell, B.; Gutteridge, J. M. C., eds.; 2a ed., Oxford University Press: Oxford, 1998, cap. 3.

6. Baynes, J. W.; Diabetes 1991, 40, 405; Alexander, R. W.; Hypertension 1995, 25, 155; Hack, V.; Breitkreutz, R.; Kinscherf, R.; Röhrer, H.; Bartsch, P.; Taut, F.; Benner, A.; Droge, W.; Blood 1998, 92, 59; Ji, L. L.; Dillon, D.; Wu, E.; Am. J. Physiol. 1990, 258, R918.

7. Alessio, H. M.; Med. Sci. Sports Exerc. 1993, 25, 218.

8. Pyne, D. B.; Aust. J. Sci. Med. Sport. 1994, 26, 49; Frankiewicz-Jozko, A.; Faff, J.; Sieradzan-Gabelska, B.; Eur. J. Appl. Physiol. Occup. Physiol. 1996, 74, 470; Tiidus, P. M.; Can. J. Physiol. Pharmacol. 1998, 76, 533.

9. Chevion, S.; Moran, D. S.; Heled, T.; Shani, Y.; Regev, G.; Abbou, B.; Berenshtein, E.; Stadtman, E. R.; Epstein, Y.; Proc. Natl. Acad. Sci. U. S. A. 2003, 100, 5119; Duthie, G. G.; Robertson, J. D.; Maughan, R. J.; Morrice, P. C.; Arch. Biochem. Biophys. 1990, 282, 78; Gleeson, M.; Robertson, J. D.; Maughan, R. J.; Clin. Sci. 1987, 73, 501; Schippinger G.; Wonisch, W.; Abuja, P. M.; Fankhauser, F.; Winklhofer-Roob, B. M.; Halwachs, G.; Eur. J. Clin. Invest. 2002, 32, 686.

10. Child, R. B.; Wilkinson, D. M.; Fallowfield, J. L.; Donnelly, A. E.; Med. Sci. Sports Exerc. 1998, 30, 1603.

11. Child, R.; Brown, S.; Day, S.; Donnelly, A.; Roper, H.; Saxton, J.; Clin. Sci. 1999, 96, 105.

12. Liu, M. L.; Bergholm, R.; Mäkimattila, S.; Lahdenperä, S.; Valkonen, M.; Hilden, H.; Yki-Järvinen, H.; Taskinen, M.; Am. J. Physiol. 1999, 276, E1083.

13. Brites, F. D.; Evelson, P. A.; Christiansen, M. G.; Nicol, M. F.; Basilico, M. J.; Wikinski, R. W.; Llesuy, S. F.; Clin. Sci. 1999, 96, 381.

14. Venditti, P.; Di Meo, S.; Arch. Biochem. Biophys. 1996, 331, 63. 
15. Powers, S. K.; Ji, L. L.; Leeuwenburg, C.; Med. Sci. Sports Exerc. 1999, $31,987$.

16. Reid, M. B.; J. Appl. Physiol. 2001, 90, 724.

17. Ji, L. L.; Exp. Biol. Med. 1999, 222, 283.

18. Sen, C. K.; J. Appl. Physiol. 1995, 79, 675.

19. Antunes-Neto, J. M.; Tese de Doutorado, Universidade Estadual de Campinas, Brasil, 2003.

20. Essig, D. A.; Nosek, T. M.; Can. J. Appl. Physiol. 1997, 22, 409.

21. Andrade, F. H.; Reid, M. B.; Allen, D. G.; Westerblad, H.; J. Physiol. 1998, 509, 565; Reid, M. B.; Khawli, F. A.; Moody, M. R.; J. Appl. Physiol. 1993, $75,1081$.

22. Zoppi, C. C.; Antunes-Neto, J.; Catanho, F. O.; Goulart, L. F.; Moura e Moura, N.; Macedo, D. V.; Rev. Paul. Educ. Fís. 2003, 17, 119.

23. Cao, G.; Prior, R. L.; Clin. Chem. 1998, 44, 1309.

24. Janaszewska, A.; Bartosz, G.; Scand. J. Clin. Lab. Invest. 2002, 62, 231.

25. Ghiselli, A.; Serafini, M.; Maiani, G.; Azzini, E.; Ferro-Luzzi, A.; Free Radical Biol. Med. 1995, 18, 29.

26. Wayner, D. D.; Burton, G. W.; Ingold, K. U.; Locke, S.; FEBS Lett. 1985, $185,33$.

27. Metsä-Ketelä, T. Em Bioluminescence and Chemiluminescence Current Status; Stanley, P.; Kricka, L., eds.; Wiley \& Sons: Chichester, 1991.

28. Glazer, A. N.; FASEB J. 1988, 2, 2487.

29. Glazer, A. N.; Meth. Enzymol. 1990, 186,161.

30. Cao, G.; Alessio, H. M.; Cutler, R.G.; Free Radical Biol. Med. 1993, 14, 303.

31. Miller, N. J.; Rice-Evans, C.; Davies, M. J.; Gopinathan, V.; Milner, A.; Clin. Sci. 1993, 84, 407.

32. Benzie, I. F. F.; Strain, J. J.; Anal. Biochem. 1996, 239, 70.
33. Mabbott, G. A.; J. Chem. Educ. 1983, 60, 697.

34. Kissinger, P. T.; Heineman, W. R.; J. Chem. Educ. 1983, 60, 702.

35. Heineman, W. R.; Kissinger, P. T. Em Laboratory Techniques in Electroanalytical Chemistry; Kissinger, P. T.; Heineman, W. R., eds.; 2a ed., Marcel Dekker, Inc.: New York, 1996, cap.3.

36. Galato, D.; Ckless, K.; Susin, M. F.; Giacomelli, C.; Ribeiro-do-Valle, R. M.; Spinelli, A.; Redox Rep. 2001, 6, 243.

37. Tan, D. X.; Manchester, L. C.; Burkhardt, S.; Sainz, R. M.; Mayo, J. C.; Kohen, R.; Shohami, E.; Huo, Y. S.; Hardeland, R.; Reiter, R. J.; FASEB J. 2001, 15, 2294.

38. Chevion, S.; Berry, E. M.; Kitrossky, N.; Kohen, R.; Free Radical Biol. Med. 1997, 22, 411.

39. Kohen, R.; Elangovan, V.; Hrbac, J.; Gati, I.; Tirosh, O.; Free Radical Biol. Med. 2000, 28, 871.

40. Chevion, S.; Roberts, M. A.; Chevion, M.; Free Radical Biol. Med. 2000, 28,860 .

41. Kohen, R.; Beit-Yannai, E.; Berry, E. M.; Tirosh, O.; Meth. Enzymol. 1999, $300,285$.

42. Motchnik, P. A.; Frei, B.; Ames, B. N.; Meth. Enzymol. 1994, 234, 269

43. Chevion, S.; Hofmann, M.; Ziegler, R.; Chevion, M.; Nawroth, P.P.; Biochem. Molec. Biol. Int. 1997, 41, 317.

44. Beit-Yannai, E.; Kohen, R.; Horowitz, M.; Trembovler, V.; Shohami, E.; J. Cereb. Blood Flow Met. 1997, 17, 273.

45. Kissinger, P. T.; Jonathan, B. H.; Adams, R. N.; Brain Res. 1973, 55, 209; Kohen, R.; Tirosh, O.; Kopolovich, K.; Exper. Geronto. 1992, 27, 161; Kohen, R.; Tirosh, O.; Gorodetsky, R.; Free Radical Res. Commun. 1992, 17, 239. 